\title{
Phenomenological Hints from a Class of String Motivated Model Constructions
}

\author{
Hans Peter Nilles \\ Bethe Center for Theoretical Physics and Physikalisches Institut der Universität Bonn, Nussallee 12, 53115 Bonn, Germany \\ Correspondence should be addressed to Hans Peter Nilles; nilles@th.physik.uni-bonn.de
}

Received 23 April 2015; Accepted 28 July 2015

Academic Editor: Michal Malinský

Copyright (C) 2015 Hans Peter Nilles. This is an open access article distributed under the Creative Commons Attribution License, which permits unrestricted use, distribution, and reproduction in any medium, provided the original work is properly cited. The publication of this article was funded by SCOAP ${ }^{3}$.

\begin{abstract}
We use string theory constructions towards the generalisation of the supersymmetric standard model of strong and electroweak interactions. Properties of the models depend crucially on the location of fields in extradimensional compact space. This allows us to extract some generic lessons for the phenomenological properties of the low energy effective action. Within this scheme we present a compelling model based on local grand unification and mirage mediation of supersymmetry breakdown. We analyse the properties of the specific model towards its possible tests at the LHC and the complementarity to direct dark matter searches.
\end{abstract}

\section{Introduction}

There are many arguments for physics beyond the $S U(3) \times$ $S U(2) \times U(1)$ standard model (SM) of strong and electroweak interaction. Unfortunately we have not seen any sign of it yet. One could hope that in the near future particle physics experiments at the LHC as well as direct (or indirect) searches for dark matter (DM) might reveal signs of physics beyond the standard model (BSM). We have to rely on theoretical prejudice in the discussion of BSM phenomena. In this paper we want to discuss the possible phenomenology of string motivated models. This is a so-called top-down approach to obtain a unified description of all interactions including gravity. It starts with a universal scheme where many things can come together: supersymmetry, extra dimensions, axions, grand unification additional singlets, additional $U(1)$ gauge bosons, and maybe much more. This is in contrast to bottom-up approaches that start with a very specific idea (e.g., supersymmetry) and work out the consequences within a minimal scheme (e.g., the minimal supersymmetric standard model (MSSM)). In the top-down approach we are not necessarily driven to such minimal schemes; the structure could be much richer.

String theories require $D=10$ (or $D=11$ ) space-time dimensions and supersymmetry for consistency. The connection to our world in $D=4$ requires the compactification of extra spatial dimensions and this leads to potentially many solutions: sometimes called the "String Landscape." While in $D=10$ the picture is simple, this is no longer true in $D=4$. This implies that many of the properties of the low energy effective action depend on details of the compactification scheme. This reduces the predictive power of string theory substantially. "String phenomenology" explores properties of the Landscape and tries to relate low energy phenomena with specific features of the fields in compactified extra dimensions. One looks for possibilities to incorporate particle physics models in a consistent way and tries to identify common properties of such models. A central aspect of string theory is supersymmetry. It is a necessity for the ultraviolet (UV) consistency of the theory. However, supersymmetry has to be partially broken in the process of compactification, but there remains the option of a $N=1$ supersymmetry down to energies as low as a TeV. This is the assumption we make in the present work. It requires new BSM physics that might be detected experimentally within the near future.

The paper will be structured as follows. In the next section we will discuss the motivation to consider supersymmetric extensions of the standard model from both the bottomup and the top-down perspective. In Section 3 we will then present string theory constructions and analyse possible lessons for SUSY model building. This will be followed by an analysis of the possible reach of experimental searches at 
the LHC given present results of the first LHC run. We will then present a specific string-inspired scenario in Section 5 and analyse its properties and possible signals for LHC as well as dark matter. We will, in particular, discuss the complementarity of collider searches and direct dark matter detection. If dark matter has its origin in supersymmetric particles we might be able to obtain an upper limit on the lightest supersymmetric particle and this might give us important hints for LHC searches. Section 6 will be devoted to conclusions and outlook.

\section{The Quest for Supersymmetry}

Supersymmetry is a necessary ingredient for any consistent string theory. However, it has to be broken in the process of compactification and a priori we do not know the breakdown scale. In models of particle physics we are confronted with the appearance of mass scales which are widely separated, as, for example, the Planck scale of $10^{18} \mathrm{GeV}$ and the weak scale in the $\mathrm{TeV}$ region. In usual quantum field theories these hierarchies of scales might be unstable because of the appearance of quantum corrections. In the standard model this concerns the stability of the mass of the fundamental scalar Higgs boson. A protection of the Higgs mass can be achieved within a supersymmetric scheme; the main motivation for supersymmetry $[4,5]$ is the stability of the weak scale: this mechanism requires new particles at the weak scale, partners of quarks, leptons, and gauge bosons that could be discovered at LHC. These new particles have influence on the evolution of the gauge coupling constants. Remarkably they lead to a situation that (within the MSSM) the gauge couplings of $S U(3), S U(2)$, and $U(1)$ meet at the grand unification scale of order of a few times $10^{16} \mathrm{GeV}$; a second strong motivation for supersymmetry is gauge coupling unification: supersymmetric model requires special care with proton stability. A solution is the postulate of a new (discrete) symmetry, matter parity in the simplest case. Such a symmetry predicts the existence of a new stable particle and this is further motivation for SUSY as it provides dark matter candidates that seem to be required by astroparticle and cosmological observations. Good candidates are neutral, weakly interacting particles, known as WIMPs. They could be subject to detection at the LHC as well as dedicated dark matter search experiments.

A further attractive property of supersymmetry from the bottom-up approach is the fact that local (gauged) supersymmetry automatically includes gravitational interactions in the form of supergravity = gauged supersymmetry: from the topdown approach this is, of course, obvious as a cornerstone of the unification of all interactions in the framework of string theory. All these arguments make lower energy (TeV) scale supersymmetry a very attractive framework for physics beyond the standard model. In this paper we will therefore assume the presence of low energy supersymmetry.

\section{String Model Building}

To learn some lessons from string theory we need to proceed to explicit model building towards the MSSM and generalisations thereof. The properties of the low energy effective action depend strongly on the process of compactification. It is not enough to know the nature of the compact manifold but we also need to know the location of the various fields on this manifold. Some of the fields might reside in the full 6-dimensional manifold (called bulk fields) while others are localized on submanifolds (so-called brane fields). We thus need knowledge about the geography of fields in extra dimensions [6]. The localized fields might be subject to different amounts of symmetry both for gauge and supersymmetry. This leads to a scheme known as local grand unification that has enhanced grand unified gauge symmetry for certain brane fields.

While there have been many attempts to construct particle physics models from string theory [7, 8] only few of them are realistic and explicit enough to analyse the questions under consideration. We therefore will concentrate on a set of models based on the so-called MiniLandscape [9-12] and its generalisations [13-23]. These constructions are based on the heterotic $E_{8} \times E_{8}$ string compactified on 6-dimensional orbifolds: the so-called heterotic braneworld [24]. The $S U(3) \times S U(2) \times U(1)$ gauge group of the standard model is a subgroup of one of the $E_{8}$ groups with a hidden intermediate $S O(10)$ local GUT structure to admit 16dimensional spinor representations for the families of quarks and leptons (along the rules of [25]).

These models have the following space-time structure: bulk fields that live in the full 10 space-time dimensions (6 dimensions in compactified space), fixed tori (fields that live in 6-dimensional space-time, i.e., two dimensions in compact space), and fixed points in compact dimensions (fields live in 4-space-time dimensions). In heterotic string theory the gauge fields are bulk fields, while matter or Higgs fields can live either in the bulk, on fixed tori, or on fixed points. Localized fields can experience different amounts of gauge symmetry depending on which point or torus they are localized on (this is the concept of local grand unification (like $S O(10)$ enhancement at some of the fixed points [11])). The relative location of matter and Higgs fields is important for the properties of the low energy effective action as it determines size of couplings and mixings in the Yukawa sector.

With the large number of realistic MSSM and generalized MSSM candidates in the MiniLandscape we can now analyse the properties of the successful models. The models typically contain additional singlets and $U(1)$ gauge symmetries.

3.1. Lessons from the MiniLandscape. There are some amazing universal properties. The first one concerns the Higgs fields: they are bulk fields, and this seems to be a generic property of the successful models. This implies that the Higgs bosons descend from gauge fields in higher dimensions and thus exhibit so-called gauge-Higgs unification. In fact, it is in general nontrivial to keep a massless Higgs pair $H, \bar{H}$ in the low energy theory as this pair is vector-like and could become heavy through a $\mu$-term, $\mu H \bar{H}$ in the superpotential. The MiniLandscape provides a solution to the $\mu$-problem. The $\mu$-term is forbidden by (approximate) discrete $R$-symmetry 
$[11,26]$, and the supersymmetric vacua are of Minkowski type. This is reminiscent of an earlier suggestion of Casas and Muñoz [27]. A $\mu$-term can be generated with broken supersymmetry and its size is therefore related to soft SUSY breaking parameters. The $R$-symmetry treats bosons and fermions differently. It descends from the Lorentz groups $S O(6)$ (subgroup of $S O(9,1)$ ) of compactified space. This is the reason why the light Higgs fields have to live in the bulk. These $R$-symmetries have to be studied in detail to understand the size of the $\mu$-term also in relation to the gravitino mass $[28,29]$.

Let us now turn to quarks and leptons. The top-quark is special as it has a mass comparable to the gauge bosons and thus a Yukawa coupling of order of the gauge coupling: so-called gauge-top unification. In the models of the MiniLandscape the top-quark has (in contrast to most of the other quarks and leptons) a nontrivial trilinear (tree level) coupling to the Higgs field. This gives us the second amazing property of the MiniLandscape. The top-quark is a bulk field as well! It is the large spatial overlap with the Higgs field that provides the large top-quark Yukawa coupling. The location of the other fields of the third family is rather model dependent. In most cases none of the additional fields has trilinear Yukawa couplings.

In contrast to the third family, the first two families live at fixed points. At these special points one typically has enhanced gauge and discrete symmetries. In the models discussed here we find a discrete $D_{4}$ family symmetry [11] (a discrete subgroup of an $S U(2)$ flavour symmetry [30]). This alleviates possible problems with flavour changing neutral currents. The first two families are (in models of the MiniLandscape) subject to an $S O(10)$ local subgroup enhancement (at leading order) at the representative fixed point. We thus see that all the important aspects of particle physics find a convincing geometrical and geographical explanation within the framework of the heterotic MiniLandscape. Basic ingredients are the gauge symmetry structure and the action of discrete symmetries [31], as the R-symmetry in the Higgs sector and the $D_{4}$ flavour symmetry. So far our discussion on the structure of supersymmetric models. The models contain possibly additional singlets and $U(1)$ gauge symmetries. The lessons described here are important hints for model building and should therefore be incorporated in bottom-up schemes as well.

3.2. Pattern of Supersymmetry Breakdown. In absence of a convincing alternative one would suggest some kind of gravity (modulus) mediation in the framework of string theory. Analysis of moduli stabilization and SUSY breakdown in type II [32-34] and heterotic theory [35] reveal the important fact that the strength of gravity mediation might even be reduced. Radiative corrections could then become important, leading to contributions that are reminiscent of anomaly mediation. Quite generically this leads to a scheme of so-called mirage mediation, a scheme of mixed modulus (gravity) mediation and anomaly mediation. Contributions of modulus mediation are typically suppressed by a factor $\log \left(M_{\text {Planck }} / m_{3 / 2}\right)$ where $m_{3 / 2}$ denotes the gravitino mass. Radiative contributions become competitive leading to the specific pattern of mirage mediation as explained in detail in [33]. This, in particular, leads to a rather interesting pattern of gaugino masses [36].

Geographical properties of fields in compactified space are important for the discussion of SUSY breakdown as well. In $D=4$ space-time dimensions we obtain $N=$ 1 supersymmetry through the process of compactification. Various subsectors, however, might experience a higher degree of supersymmetry. Bulk fields, for example, live on the six-dimensional torus (underlying the (flat) orbifold). Torus compactification would lead to $N=4$ extended supersymmetry in $D=4$. The suppression of the contribution of modulus mediation for bulk fields as described above could be attributed to the presence of this extended supersymmetry (at leading order). Fields on a fixed torus might experience an approximate $N=2$ supersymmetry while fields at fixed points feel only $N=1$ at leading order. Therefore the SUSYprotection depends on the location of the fields. We would thus expect a hierarchy of soft mass terms. They would be large for the masses of the first two generations and relatively suppressed for fields of the third generation as well as the gauginos. Less is known concerning the discussion of the $\mu$-term as this is allowed by supersymmetry. Of course, we have a protection with an $R$-symmetry at the level of the soft SUSY breaking terms $[11,26]$, but generically we do not know whether this corresponds to the values of the gaugino masses or the (larger) masses of the scalars of the first two generations. As $\mu$ determines the mass of the higgsinos (a potential dark matter candidate) it is important to spend more efforts on that issue (see, e.g., [29]). Apart from the $\mu$ term we have, however, a pretty convincing pattern of SUSY breakdown relevant for the phenomenological properties of the models to confront LHC data. It should be stressed that these discussions took place before LHC came into operation and have not been influenced by LHC results. Of course, now we have to analyse whether the scheme is compatible with the experimental results and find out what we can learn from there. We are still in the dark and need some hints from experiment.

\section{Experimental Situation after the First LHC Run}

Unfortunately there is no sign for either supersymmetry or any physics beyond the standard model. Still we have some results from LHC searches that have to be taken into account. The Higgs boson has been found with a mass of $125 \mathrm{GeV}$ $[37,38]$. This is compatible with the MSSM but rather close to the upper limit of, let us say, $130 \mathrm{GeV}$. A higher Higgs mass would have ruled out SUSY (or the MSSM) and so LHC failed to rule out SUSY. Within the MSSM this large mass of $m_{\text {Higgs }}$ requires SUSY partners at a high scale. In this regime the MSSM Higgs is rather similar to the Higgs in the standard model and SUSY partners are heavy. This is not necessarily true for models beyond the MSSM. In models with additional singlets we might have a nontrivial mixing of the Higgs bosons. If we would have known the mass of the Higgs boson before LHC came into operation we would have probably been less optimistic about SUSY searches there. 
In that sense, the absence of any sign of SUSY particles at the LHC is not yet incompatible even within the MSSM. The question is as follows: Will the LHC with its energy reach be able to answer the question of any (TeV-scale) physics beyond the standard model? We do not really know. Answers are highly model dependent. So let us go through the basic arguments for SUSY. First there is the stability of the weak scale that requires new states in its vicinity. Typically we could thus look at the question of the amount of fine-tuning needed to explain the absence of light SUSY states. But these finetuning arguments have to be taken with care, especially in absence of any other reason for the stability of the weak scale that requires new states in its vicinity.

A second argument is the question of gauge coupling unification present in the MSSM but absent in the SM. This requires new states and we will discuss this issue later in detail. So there remains, as third argument, the solution of the dark matter problem via a SUSY-WIMP. If we consider a WIMP with standard thermal abundance we should be able to get an upper limit on the mass of such a particle. There remains the question of whether the LHC will cover the full range of these possibilities. In that sense there is hope that searches at the next LHC run as well as direct and indirect detection experiments for dark matter might cover the full range of these possibilities (including precision experiments of phenomena like $g-2$, flavour violations, or precision Higgs physics).

Still, the absence of any experimental signal for physics beyond the standard model restricts the parameter space of supersymmetric models considerably. Naive expectations for large missing energy signals have not been seen yet. Simple models are not ruled out at the cost of shifting the SUSY masses (e.g., the gluino mass) to rather high values beyond a TeV. Further restrictions arise when one considers the energy content of the universe. Many models lead to an overabundance of WIMP (e.g., bino) dark matter and need modifications. So we are stuck with our theoretical ideas. We urgently need further impact from experiment. Let us hope that the next run of the LHC provides information of physics beyond the standard model. Still there remains the question of whether LHC has the energy reach to discover supersymmetry. We have to wait and see. Meanwhile we have to work out specific examples in detail that might be testable with the next run.

\section{A Representative Scheme}

It is impossible to make general predictions based on $\mathrm{TeV}$ scale supersymmetry. Let us therefore discuss a specific example which is motivated from the string-inspired discussion presented earlier. Even there we need to make some more assumptions to narrow down the various possibilities.

We make three explicit assumptions:

(i) We assume a supersymmetric model with new particles connected to the weak scale.

(ii) Dark matter should be given by a supersymmetric WIMP (as, e.g., a neutralino). This might lead to an upper limit on the mass of some of the SUSY particles if we require the correct dark matter abundance.

(iii) We assume (precision) gauge coupling unification. Again this might require new states beyond the standard model within the energy reach of LHC.

We should stress that these are ad hoc assumptions which are not necessarily predicted from the general theoretical perspective, but they seem to be a reasonable starting point to analyse the properties of these string-inspired models.

5.1. The SUSY Mass Scale. The assumption made above can tell us indirectly something about the mass scale of supersymmetric particles [39]. This is pretty obvious for the SUSY-WIMP-interpretation of dark matter which we discuss later in detail. But it is also true for the assumption of grand unification. Within our scheme, SUSY partners of standard model particles (in the $\mathrm{TeV}$ range) have to provide the necessary thresholds. Let us parametrize the SUSY scale by a single effective mass scale $M_{\text {SUSY }}$. The threshold for the evolution of the gauge coupling constants is then given by

$$
\frac{1}{g_{i, \mathrm{Thr}}^{2}}=\frac{b_{i}^{\mathrm{MSSM}}-b_{i}^{\mathrm{SM}}}{8 \pi^{2}} \ln \left(\frac{M_{\mathrm{SUSY}}}{M_{Z}}\right),
$$

where $b_{i}^{\mathrm{SM}}\left(b_{i}^{\mathrm{MSSM}}\right)$ stand for the beta function coefficients of the (supersymmetric) standard model. If all supersymmetric partners have a common mass $M$, then $M_{\text {SUSY }}=M$. We define $M_{\mathrm{GUT}}$ as the scale where the coupling constants $g_{1}$ and $g_{2}$ meet. The precision of gauge coupling unification can then be parametrized by

$$
\epsilon_{3}=\frac{g_{3}^{2}\left(M_{\mathrm{GUT}}\right)-g_{1,2}^{2}\left(M_{\mathrm{GUT}}\right)}{g_{1,2}^{2}\left(M_{\mathrm{GUT}}\right)} .
$$

The value of $\epsilon_{3}$ as a function of $M_{\text {SUSY }}$ is shown in Figure 1. It shows that precision unification gives a SUSY scale of 2$3 \mathrm{TeV}$. This appears to be rather large, given the energy reach of LHC. Of course, there might be other thresholds at higher mass scales that might modify this result to lower values of $M_{\text {SUSY }}$, but there is no a priori reason to assume this. Does this mean that all SUSY partners have to be above this scale? (Un)fortunately not. Different particles affect the evolution of coupling constants differently and we have to compute the effective scale $M_{\text {SUSY }}$ explicitly for a given model. Let us assume that squark and slepton masses for a given family are universal (in complete grand unified multiplets). Only "split" multiplets have a nontrivial effect and we obtain for the effective scale [40]

$$
M_{\mathrm{SUSY}}=\frac{m_{\widetilde{W}}^{32 / 19} m_{\widetilde{h}}^{12 / 19} m_{H}^{3 / 19}}{m_{\widetilde{g}}^{28 / 19}},
$$

with $m_{\tilde{g}}, m_{\widetilde{w}}, m_{\tilde{h}}$, and $m_{H}$ as the mass for the gluino, wino, higgsino, and heavy Higgs, respectively. From this formula we see that we cannot deduce an upper limit for the mass of the lightest supersymmetric partner. Increasing the mass of the gluino leads to a smaller value of $M_{\text {SUSY }}$. On the other 


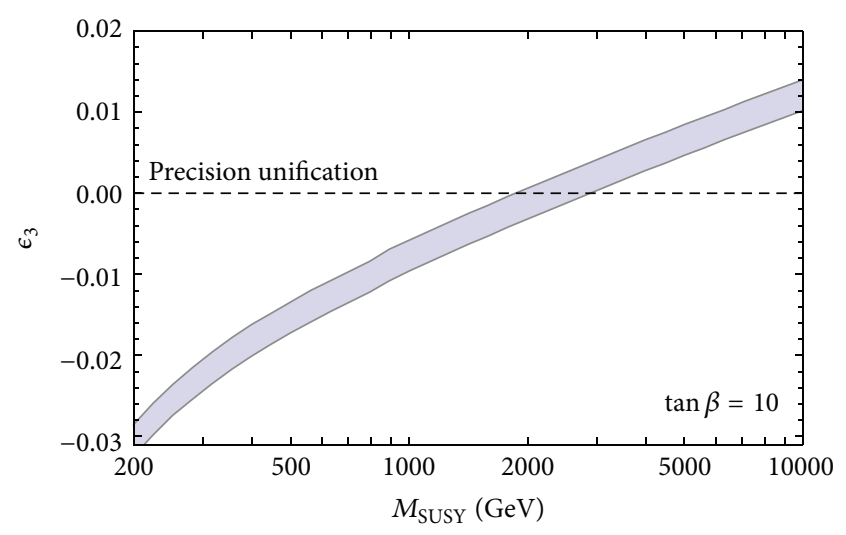

FIGURE 1: The prediction of grand unification for the effective scale $M_{\text {SUSY }}$. The width of the band represents the $1 \sigma$ experimental error in $\alpha_{s}\left(M_{Z}\right)$.

hand we also see that a large value of $M_{\text {SUSY }}$ might still be compatible with rather small masses of some supersymmetric particles in the few-hundred $\mathrm{GeV}$ range.

5.2. Mirage Mediation. Explicit discussion of supersymmetry breakdown in type IIB and heterotic string theory have revealed a specific scheme known as mirage mediation [41]. It is a combination of modulus mediation and anomaly mediation. The scheme is explained in detail, for example, in [33, 42]. The contribution of modulus mediation is suppressed by a factor $\log \left(M_{p l} / m_{3 / 2}\right) \sim 4 \pi^{2}$ so that radiative corrections become competitive. One of the specific properties of the scheme is a compressed spectrum of gaugino masses [36]. This has several important consequences:

(i) Gluinos produced at LHC will predominantly decay in neutralinos with a missing energy signal. This signal will be suppressed when the mass splitting of the gauginos is small. LHC search for missing energy will thus be less efficient than previously expected.

(ii) The usual fine-tuning problem of the weak scale is suppressed (compared to other scenarios) because the gluino mass is suppressed with respect to other neutralinos [43].

(iii) It allows the implementation of precision gauge unification in a natural way [39].

(iv) With an ultracompressed spectrum of (nearly degenerate) gauginos potential problems of the thermal relic abundance can be solved via coannihilations [39].

Mirage mediation leads to a specific spectrum: scalar partners of quarks and leptons with masses of order $m_{3 / 2}$ in the multi-TeV range, $A$-parameters, gaugino, and Higgsino masses in the $\mathrm{TeV}$ range and a compressed gaugino spectrum. These properties and the influence of precision gauge unification (PGU) have been discussed explicitly in [39] where we constructed a large sample of models and compared it to results of LHC search. We recall some of the results here and

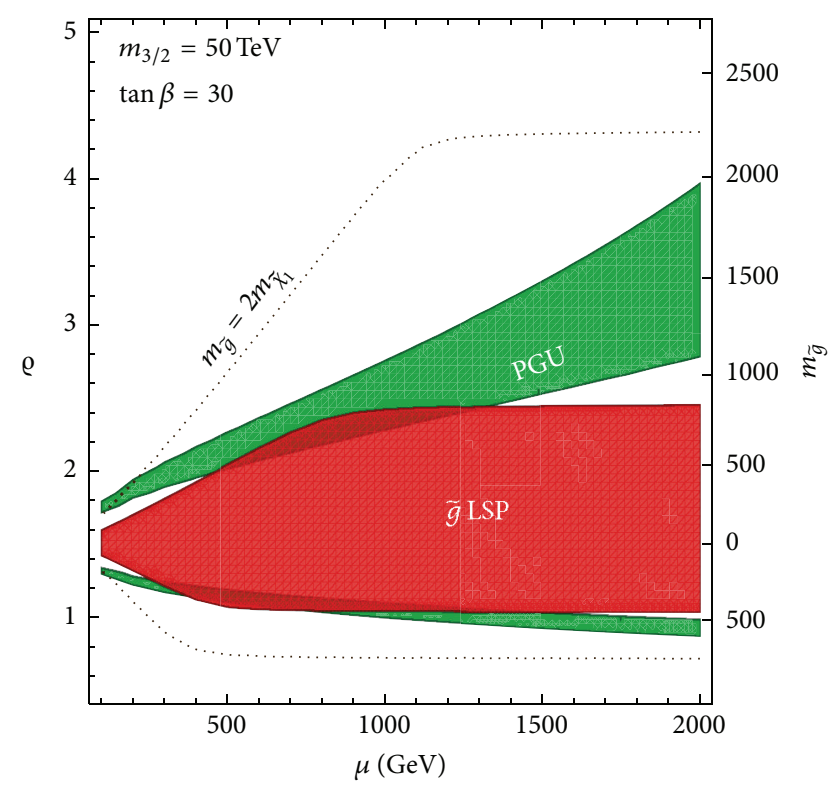

Figure 2: Parameter scan in the $(\mu, \varrho)$-plane for fixed $m_{3 / 2}=$ $50 \mathrm{TeV}$. The regions where the gauge couplings unify within the experimental error on the strong coupling are shown in green. In the upper region, the lightest neutralino is predominantly of binotype, where the lower one corresponds to a wino-like LSP. The red region exhibits a gluino LSP. The dotted contour indicates where the mass ratio between gluino and LSP becomes two.

discuss the question of dark matter candidates in more detail. We define the gaugino masses as

$$
M_{i}=\frac{m_{3 / 2}}{16 \pi^{2}}\left(\varrho+b_{i}^{\mathrm{MSSM}} g^{2}\right),
$$

such that $\varrho$ parametrizes the contribution from modulus mediation. This leads to

$$
M_{1}: M_{2}: M_{3}=(\varrho+3.3): 2(\varrho+0.5): 6(\varrho-1.5) .
$$

Pure modulus mediation would lead to $M_{1}: M_{2}: M_{3}=1$ : $2: 6$ at a low scale. To reach an effective SUSY scale $M_{\text {SUSY }} \approx$ $2 \mathrm{TeV}$ would then lead to a higgsino mass

$$
m_{\tilde{h}} \simeq 20 \mathrm{TeV}\left(\frac{\mathrm{TeV}}{m_{1 / 2}}\right)^{1 / 3}\left(\frac{\mathrm{TeV}}{m_{H}}\right)^{1 / 4}
$$

and thus a large value for $\mu \sim m_{\tilde{h}}$. This requires a large amount of fine-tuning to obtain a low value of the mass of the Higgs boson. The large value of $\mu$ can be directly understood from formula (3). If the mass of the gluino is much larger than the mass of the wino, this discrepancy has to be compensated by a large value of $m_{\tilde{h}}$. In the case of mirage mediation with a compressed spectrum the situation is quite different. The contributions from gluino and wino essentially cancel in formula (3) and gauge unification requires a smaller value of the higgsino mass and thus $\mu$. This is nicely illustrated in Figure 2. The two green regions are consistent with the requirement of (precision) gauge coupling unification. In the upper region (larger $\varrho$ ) the bino is the lightest SUSY particle 
(LSP), whereas in the lower one we predominantly have a wino WIMP. The red region has a gluino LSP and is therefore disfavoured. We see from Figure 2 that a SUSY scale $M_{\text {SUSY }} \approx$ $2 \mathrm{TeV}$ can be obtained with a $\mu$-parameter in the $\mathrm{TeV}$ range. Thus the fine-tuning of the weak scale is less severe than in the case of uncompressed gaugino mass spectra.

5.3. Constraints from LHC. To confront the scheme with present LHC results we have generated a large data sample of models within the favoured regions of Figure 2. Details are explained in [39]. As input we have chosen parameters randomly in the intervals

$$
\begin{aligned}
\mu & =0.1-2 \mathrm{TeV}, \\
\varrho & =0.5-30, \\
m_{3 / 2} & =\frac{40-200 \mathrm{TeV}}{\varrho}, \\
\tan \beta & =10-50 .
\end{aligned}
$$

The ranges of $\varrho$ and $m_{3 / 2}$ are correlated and yield a gravity mediated contribution to the gauging masses of $0.25-$ $1.25 \mathrm{TeV}$. A scatter plot with successful gauge unification models in the gluino and LSP mass plane is shown in Figure 3. The grey dots represent the individual models. To guide the eye we have included representative limits on the gluino mass from LHC searches by ATLAS [1] and CMS [2]. Both limits are not strictly applicable at this point and should be replaced by results of dedicated searches for the models under consideration. More than $90 \%$ of the benchmark points fulfil $m_{\tilde{\chi}_{1}}>0.5 m_{\tilde{g}}$. The strong compression of gaugino masses makes it more difficult to detect the gluino here than in ordinary SUSY schemes as, for example, the CMSSM. Still with the next run of the LHC it should be possible to cover the full range of small gluino masses up to "kinematic limit," depending on beam energy and collected luminosity [44].

\subsection{Constraints from Dark Matter Relic Abundance. More} constraints on the models could come from the requirement of the correct thermal relic abundance of the LSP as a candidate for dark matter. Prime contenders are the wino, the bino, and the higgsino. Due to its small annihilation cross section, the bino density from thermal production typically exceeds the observed dark matter density by far. Higgsinos, on the other hand, undergo efficient annihilations into gauge bosons or third-generation quarks, and coannihilations with the charged higgsino further enhance their cross section. Thus, the relic density of the higgsino LSP might typically be below the required dark matter density. In mirage mediation, the gaugino masses, while nonuniversal at the large scale, lead to a highly compressed spectrum at the weak scale as a consequence of the requirement of gauge coupling unification. This nearly degenerate spectrum enhances the possibility for coannihilation favourable to reduce the bino mass density in this mixed scheme. Figure 4 illustrates possible restrictions from the correct relic density. For a given value of the gravitino mass (here $50 \mathrm{TeV}$ ) the constraint is fulfilled in the blue region. We can identify two specific

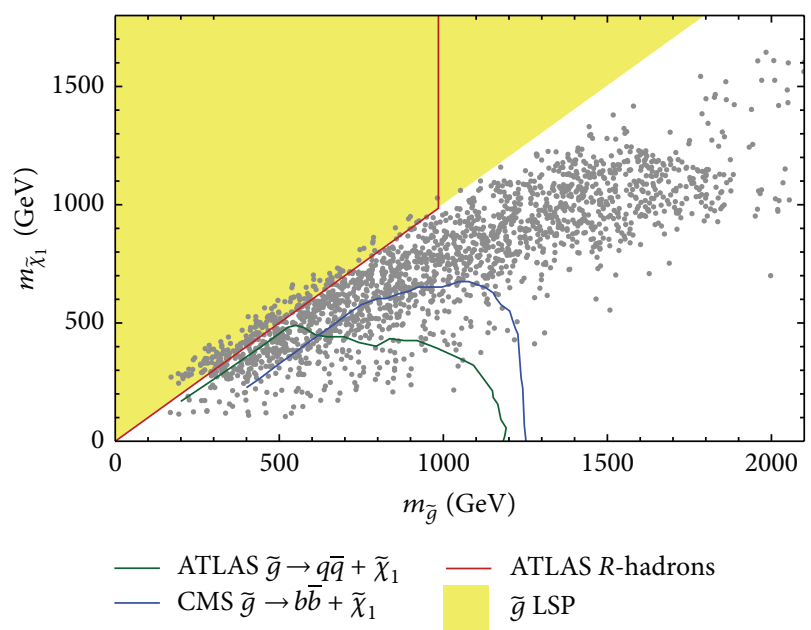

FIGURE 3: Parameter points with successful gauge coupling unification (gray). Also shown are representative constraints on the gluino mass for the decay modes $\tilde{g} \rightarrow q \bar{q}+\widetilde{\chi}_{1}$ and $\widetilde{g} \rightarrow b \bar{b}+\widetilde{\chi}_{1}$ by ATLAS [1] and CMS [2]. The yellow region features a gluino LSP which is constrained by the ATLAS search for stable $R$-hadrons.

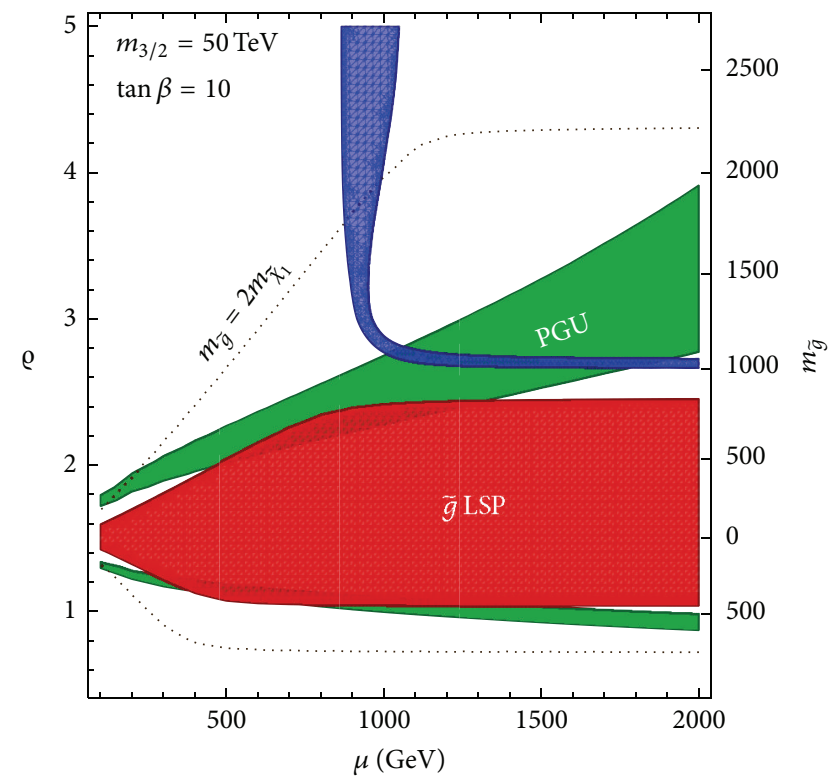

Figure 4: Parameter scan in the $(\mu, \varrho)$-plane for fixed $m_{3 / 2}=$ $50 \mathrm{TeV}$, as described earlier in Figure 2. The blue region identifies the parameters that are compatible with the correct dark matter abundance. The vertical region around $\mu \sim 1$ corresponds to a higgsino LSP, while the horizontal strip corresponds to (mixed) bino LSP, here at a gluino mass around $1 \mathrm{TeV}$. The gluino mass scales proportional to the gravitino mass.

cases: higgsino dark matter (vertical strip) with $\mu \sim 1 \mathrm{TeV}$ and a coannihilation strip (horizontal) for a fixed gluino mass (which, however, varies with the gravitino mass). The consequences of this constraint on our model sample are shown in Figure 5. Grey dots do not give the correct relic abundance, black dots refer to the bino coannihilation strip, and the purple ones refer to a higgsino LSP. There are, in 


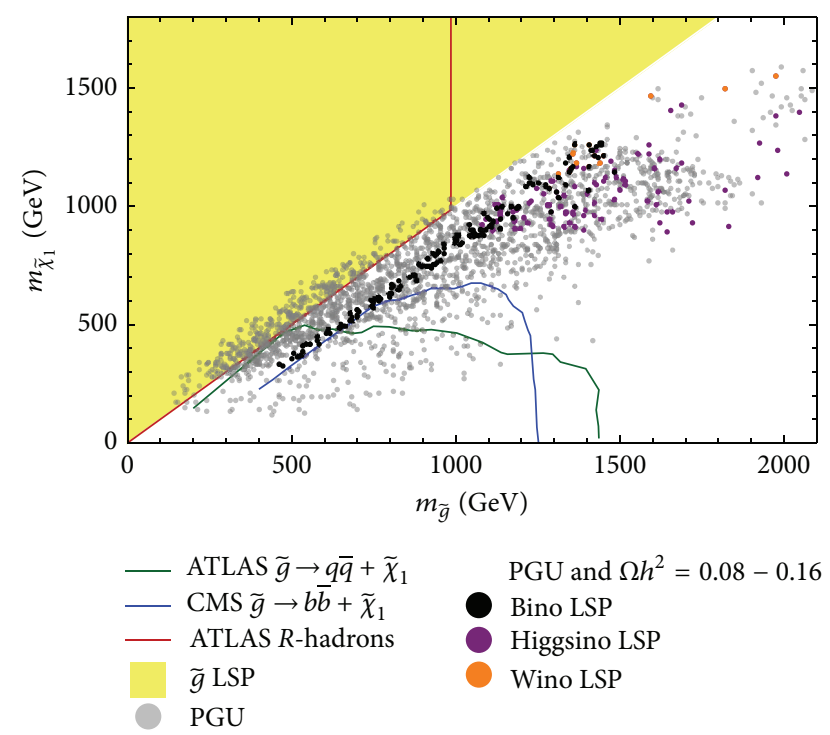

FIgURE 5: This is the same as Figure 3 but with the information on the dark matter candidates and abundance. The grey dots do not provide the correct relic density. Black dots correspond to an LSP that is predominantly bino, while purple (orange) dots correspond to higgsino (wino) LSP. The black dots are approximately aligned in a "coannihilation strip."

addition, a few dots (in orange) corresponding to a wino LSP (in case that mirage mediation is dominated by the anomaly contribution corresponding to the lower green region in Figure 2). Most of the favoured models should be within the range of the next LHC run. Particularly the black dots in the coannihilation strip should be testable in the near future, while higgsino and wino LSPs might be out of reach in some of the cases.

5.5. Complementarity of Searches. Fortunately there are other observations that can help in clarifying the situation: experiments for direct dark matter detection. WIMP candidates can be found through their interaction with nucleons. The cross sections of binos, winos, and higgsinos differ quite significantly. In the scheme discussed here, the cross section of the lightest neutralino with nucleons is dominated by the exchange of the light Higgs boson (as the other scalars are in the multi-TeV range). The coupling of the LSP to the Higgs boson is proportional to the gaugino-higgsino mixing angle; it vanishes in the limit of a pure state. The neutralinoproton cross section $\sigma_{p}$ for our benchmark points is shown in Figure 6. We find a cross section between $10^{-43} \mathrm{~cm}^{2}$ and $10^{-48} \mathrm{~cm}^{2}$. Direct detection experiments as, for example, LUX [3] have just started to probe this regime of cross sections. Experiments of the next generation should be able to test a significant fraction of the benchmark points.

For the specific scheme under consideration we observe a complementarily of direct searches for dark matter with those of the LHC. This is (also in more general cases [45]) a rather fortunate situation. The black dots ((mixed) bino LSP (when we denote the states by wino, bino, and higgsino LSP

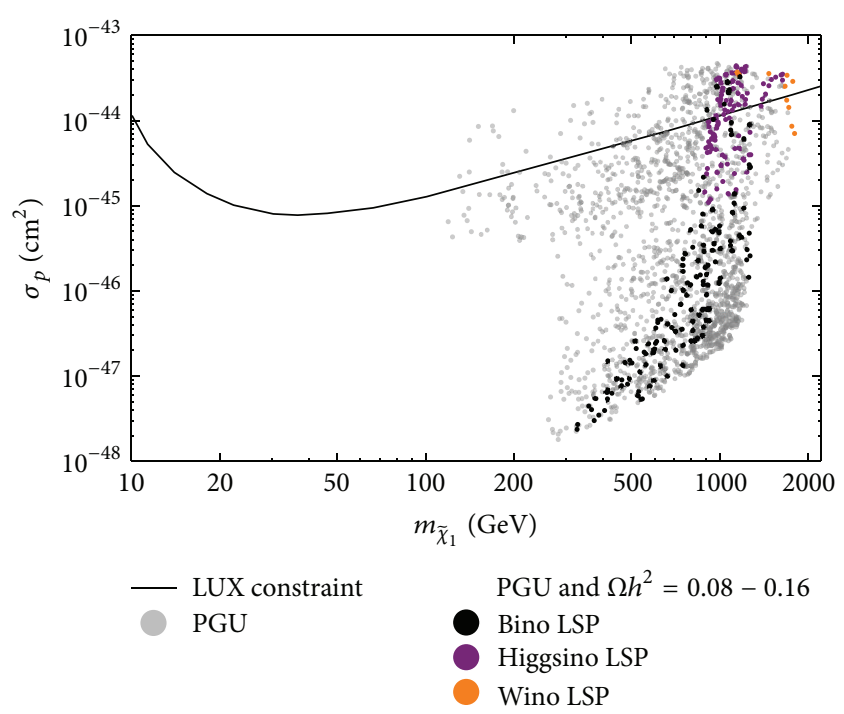

FIGURE 6: Neutralino-proton cross section for the benchmark points with successful gauge coupling unification. The colour coding is the same as in Figure 5. Models denoted by grey dots do not give the correct relic abundance for dark matter. Black, purple, and orange dots correspond to WIMP candidates that are predominantly bino, higgsino, or wino. The current limit from the LUX direct dark matter search [3] is also shown. The latter is valid if the lightest neutralino accounts for all dark matter in the universe.

we identify the dominant component, as the candidates that satisfy the constraints are usually mixed states)) are difficult to detect directly, but according to Figure 5 they might be within the reach of the upcoming run of the LHC. On the other hand, the wino and higgsino WIMP candidates are easier to see in direct detection experiments. If we look at Figure 6, we observe that many of the purple and orange dots are already ruled out. So this region of the parameter space that is possibly beyond the reach of LHC can be tested by direct dark matter detection experiments. In that sense it is likely that our benchmark scheme can be tested within the not so distant future.

\section{Conclusions}

As we have seen it is a long way from string theory via supersymmetric extensions of the standard model to LHC physics. To test these ideas we need consistent string theory constructions that allow explicit determination of spectrum and interactions to be confronted with the data. At this point only the models of the heterotic MiniLandscape satisfy both criteria. Given these models we can try to extract some generic properties from the successful supersymmetric candidate models. The origin of these lessons comes from the geographic localization of fields in compactified extradimensional space. A coherent picture emerges; Higgs and top multiplets live in the bulk. This provides a solution to the $\mu$ problem with an $R$-symmetry as well as a large value for the Yukawa coupling of the top-quark (to be consistent with socalled gauge-Yukawa unification). The multiplets of the first 
two families are located at fixed points in extradimensional space. They enjoy enhanced gauge and discrete symmetries that alleviate the flavour problem. A slight breakdown of these symmetries provides a small parameter (originated from a Fayet-Iliopoulos term) that could explain the hierarchies of quark and lepton masses as well as the $\mu$-parameter. We expect these properties (derived from the heterotic string theory) to be of more general validity and should also manifest themselves in constructions based on type I, type II, M-, or F-theory.

In the discussion of SUSY breakdown we can identify a rather generic scheme: mirage mediation. It has been found in both type IIB and heterotic theory and is a consequence of the mechanism to obtain a small value of the vacuum energy (compared to the scale of the gravitino mass). The scheme is characterized by two scales for the soft terms separated by a factor $\log \left(M_{\text {Planck }} / m_{3 / 2}\right)$. Gaugino masses and $A$-parameters tend to be at the $\mathrm{TeV}$ scale, while gravitino mass and scalar masses are generically at a higher scale. A second characteristic property of the mirage scheme is the possibility of a compressed spectrum of the gaugino masses. It leads to hidden SUSY at the LHC and allows for the correct thermal relic density of the LSP dark matter candidate. Within this scheme we could identify another important result concerning scalar masses, determined by the localization properties of the corresponding fields with a potential protection through extended supersymmetry. Localized fields as, for example, the scalar partners of quarks and leptons of the first two families only feel $N=1$ SUSY and would be as heavy as the gravitino. Fields at fixed tori or the bulk feel a hidden $N=2$ or $N=4$ SUSY and have suppressed masses comparable to those of the gaugino masses. It is this interplay of symmetries that leads to very specific properties of the spectrum of superpartners. The scheme is still consistent with all known experimental data. A large part of the parameter space is within the kinematical reach of the LHC at $13 \mathrm{TeV}$. This is partially due to the fact that those models that are beyond the reach of the LHC might be ruled out through direct dark matter detection experiments. The next run of the LHC will hopefully be able to test these ideas. Independent of the discussion here, it is obvious that we urgently need new experimental data to clarify the nature of potential physics beyond the standard model. Theoretically we have tried all we could do. There are many well-motivated models that have been analysed, but there are no real predictions. We need help from experimental data.

\section{Conflict of Interests}

The author declares that there is no conflict of interests regarding the publication of this paper.

\section{Acknowledgments}

The author would like to thank Rolf Kappl and Martin Winkler for very useful discussions as well as the preparation of the figures. This work was partially supported by the SFBTransregio TR33 "The Dark Universe" (Deutsche Forschungsgemeinschaft).

\section{References}

[1] ATLAS Collaboration, "Search for squarks and gluinos with the ATLAS detector using final states with jets and missing transverse momentum and $5.8 \mathrm{fb}^{-1}$ of $\sqrt{s}=8 \mathrm{TeV}$," ATLASCONF-2012-109, ATLAS-COM-CONF-2012-140.

[2] CMS Collaboration, Search for Supersymmetry in pp collisions at $8 \mathrm{TeV}$ in events with a single lepton, multiple jets and b-tags, CMS-PAS-SUS-13-007.

[3] D. S. Akerib, H. M. Araújo, X. Bai et al., "First results from the LUX dark matter experiment at the Sanford underground research facility," Physical Review Letters, vol. 112, Article ID 091303, 2014.

[4] H. P. Nilles, "Supersymmetry, supergravity and particle physics," Physics Reports, vol. 110, no. 1-2, pp. 1-162, 1984.

[5] H. E. Haber and G. L. Kane, "The search for supersymmetry: probing physics beyond the standard model," Physics Reports, vol. 117, pp. 75-263, 1985.

[6] H. P. Nilles and P. K. S. Vaudrevange, "Geography of fields in extra dimensions: string theory lessons for particle physics," Modern Physics Letters A, vol. 30, no. 10, Article ID 1530008, 2015.

[7] L. E. Ibanez and A. M. Uranga, String Theory and Particle Physics: An Introduction to String Phenomenology, Cambridge University Press, Cambridge, UK, 2012.

[8] K.-S. Choi and J. E. Kim, Quarks and Leptons from Orbifolded Superstring, vol. 696 of Lecture Notes in Physics, Springer, Berlin, Germany, 2006.

[9] O. Lebedev, H. P. Nilles, S. Raby et al., "A mini-landscape of exact MSSM spectra in heterotic orbifolds," Physics Letters B, vol. 645, no. 1, pp. 88-94, 2007.

[10] O. Lebedev, H.-P. Nilles, S. Raby et al., "Low energy supersymmetry from the heterotic string landscape," Physical Review Letters, vol. 98, Article ID 181602, 2007.

[11] O. Lebedev, H. Nilles, S. Raby et al., "Heterotic road to the MSSM with $R$ parity," Physical Review D, vol. 77, Article ID 046013, 2007.

[12] O. Lebedev, H. P. Nilles, S. Ramos-Sánchez, M. Ratz, and P. K. S. Vaudrevange, "Heterotic mini-landscape (II): completing the search for MSSM vacua in a $\mathrm{Z}_{6}$ orbifold," Physics Letters B, vol. 668, no. 4, pp. 331-335, 2008.

[13] T. Kobayashi, S. Raby, and R.-J. Zhang, "Constructing 5d orbifold grand unified theories from heterotic strings," Physics Letters B, vol. 593, no. 1-4, pp. 262-270, 2004.

[14] T. Kobayashi, S. Raby, and R. J. Zhang, "Searching for realistic $4 \mathrm{~d}$ string models with a Pati-Salam symmetry. Orbifold grand unified theories from heterotic string compactification on a $\mathrm{Z}_{6}$ orbifold," Nuclear Physics B, vol. 704, pp. 3-55, 2005.

[15] W. Buchmüller, K. Hamaguchi, O. Lebedev, and M. Ratz, "Supersymmetric standard model from the heterotic string," Physical Review Letters, vol. 96, Article ID 121602, 2006.

[16] W. Buchmüller, K. Hamaguchi, O. Lebedev, and M. Ratz, "Supersymmetric standard model from the heterotic string (II)," Nuclear Physics B, vol. 785, no. 1-2, pp. 149-209, 2007.

[17] M. Blaszczyk, S. G. Nibbelink, M. Ratz, F. Ruehle, M. Trapletti, and P. K. S. Vaudrevange, "A $\mathrm{Z}_{2} \times \mathrm{Z}_{2}$ standard model," Physics Letters B, vol. 683, pp. 340-348, 2010.

[18] J. E. Kim and B. Kyae, "Flipped $S U(5)$ from $Z_{12-I}$ orbifold with Wilson line," Nuclear Physics B, vol. 770, no. 1-2, pp. 47-82, 2007.

[19] J. E. Kim and B. Kyae, "String MSSM through flipped SU(5) from $\mathrm{Z}_{12}$ orbifold," http://arxiv.org/abs/hep-th/0608085. 
[20] J. E. Kim, J. H. Kim, and B. Kyae, "Superstring standard model from $\mathbb{Z}_{12-I}$ orbifold compactification with and without exotics, and effective R-parity," Journal of High Energy Physics, vol. 2007, no. 6, article 034, 2007.

[21] D. K. M. Peña, H. P. Nilles, and P.-K. Oehlmann, "A zip-code for quarks, leptons and Higgs bosons," Journal of High Energy Physics, vol. 2012, no. 12, article 024, 2012.

[22] S. G. Nibbelink and O. Loukas, "MSSM-like models on $Z_{8}$ toroidal orbifolds," Journal of High Energy Physics, vol. 2013, no. 12, article 044, 2013.

[23] H. P. Nilles, S. Ramos-Sanchez, M. Ratz, and P. K. S. Vaudrevange, "From strings to the MSSM," The European Physical Journal C, vol. 59, no. 2, pp. 249-267, 2009.

[24] S. Förste, H. P. Nilles, P. Vaudrevange, and A. Wingerter, "Heterotic brane world," Physical Review D, vol. 70, Article ID 106008, 2004.

[25] H. P. Nilles, "Five golden rules for superstring phenomenology," in Proceedings of the 10th International Symposium on Particles, Strings and Cosmology, August 2004.

[26] R. Kappl, H. P. Nilles, S. Ramos-Sánchez, M. Ratz, K. SchmidtHoberg, and P. K. Vaudrevange, "Large hierarchies from approximate R symmetries," Physical Review Letters, vol. 102, no. 12, Article ID 121602, 2009.

[27] J. A. Casas and C. Muñoz, "A natural solution to the $\mu$ problem," Physics Letters B, vol. 306, no. 3-4, pp. 288-294, 1993.

[28] H. M. Lee, S. Raby, M. Ratz et al., "A unique $\mathbb{Z}_{4}^{R}$ symmetry for the MSSM," Physics Letters B, vol. 694, no. 4-5, pp. 491-495, 2011.

[29] M. Ratz and P. K. S. Vaudrevange, "Singlet extensions of the MSSM with $\mathrm{Z}_{4} \mathrm{R}$ symmetry," http://arxiv.org/abs/1502.07207.

[30] T. Kobayashi, H. P. Nilles, F. Plöger, S. Raby, and M. Ratz, "Stringy origin of non-Abelian discrete flavor symmetries," Nuclear Physics B, vol. 768, no. 1-2, pp. 135-156, 2007.

[31] H. P. Nilles, M. Ratz, and P. K. S. Vaudrevange, "Origin of family symmetries," Fortschritte der Physik, vol. 61, no. 4-5, pp. 493506, 2013.

[32] K. Choi, A. Falkowski, H. P. Nilles, M. Olechowski, and S. Pokorski, "Stability of flux compactifications and the pattern of supersymmetry breaking," Journal of High Energy Physics, vol. 2004, no. 11, article 076, 2004.

[33] K. Choi, A. Falkowski, H. P. Nilles, and M. Olechowski, "Soft supersymmetry breaking in KKLT flux compactification," Nuclear Physics B, vol. 718, no. 1-2, pp. 113-133, 2005.

[34] O. Lebedev, H. P. Nilles, and M. Ratz, "de Sitter vacua from matter superpotentials," Physics Letters B, vol. 636, no. 2, pp. 126-131, 2006.

[35] V. Löwen and H. P. Nilles, "Mirage pattern from the heterotic string," Physical Review D, vol. 77, Article ID 106007, 2008.

[36] K. Choi and H. P. Nilles, “The gaugino code," Journal of High Energy Physics, vol. 2007, no. 4, article 6, 2007.

[37] G. Aad, T. Abajyan, B. Abbott et al., "Observation of a new particle in the search for the Standard Model Higgs boson with the ATLAS detector at the LHC," Physics Letters B, vol. 716, no. 1, pp. 1-29, 2012.

[38] S. Chatrchyan, V. Khachatryan, A. M. Sirunyan et al., "Observation of a new boson at a mass of $125 \mathrm{GeV}$ with the CMS experiment at the LHC," Physics Letters B, vol. 716, no. 1, pp. 30-61, 2012.

[39] S. Krippendorf, H. P. Nilles, M. Ratz, and M. W. Winkler, "Hidden SUSY from precision gauge unification," Physical Review D, vol. 88, Article ID 035022, 2013.
[40] M. Carena, S. Pokorski, and C. E. M. Wagner, "On the unification of couplings in the minimal supersymmetric standard model," Nuclear Physics B, vol. 406, no. 1-2, pp. 59-89, 1993.

[41] O. Loaiza-Brito, J. Martin, H. P. Nilles, and M. Ratz, " $\log \left(M_{P l} / m_{3 / 2}\right)$," AIP Conference Proceedings, vol. 805, no. 1, pp. 198-204, 2005.

[42] S. Krippendorf, H. P. Nilles, M. Ratz, and M. W. Winkler, "The heterotic string yields natural supersymmetry," Physics Letters $B$, vol. 712, no. 1-2, pp. 87-92, 2012.

[43] O. Lebedev, H. P. Nilles, and M. Ratz, "A note on fine-tuning in mirage mediation," in Proceedings of the Symposium in Honor of Gustavo C. Branco: CP Violation and the Flavor Puzzle, Lisbon, Portugal, July 2005.

[44] ATLAS collaboration, "Expected sensitivity studies and quark searches," ATLASNOTE ATL-PHYS-PUB-2015-005, 2015.

[45] G. B. Gelmini, "TASI 2014 lectures: the hunt for dark matter," http://arxiv.org/abs/1502.01320. 

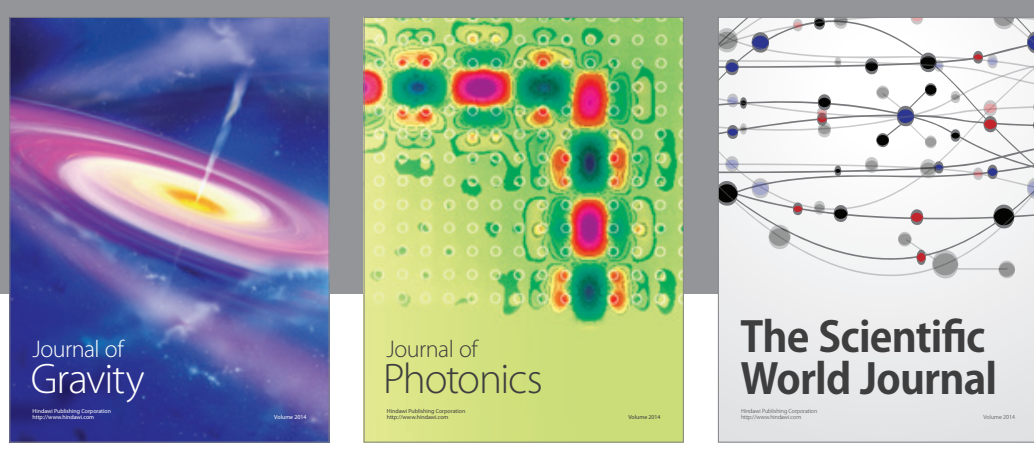

The Scientific World Journal
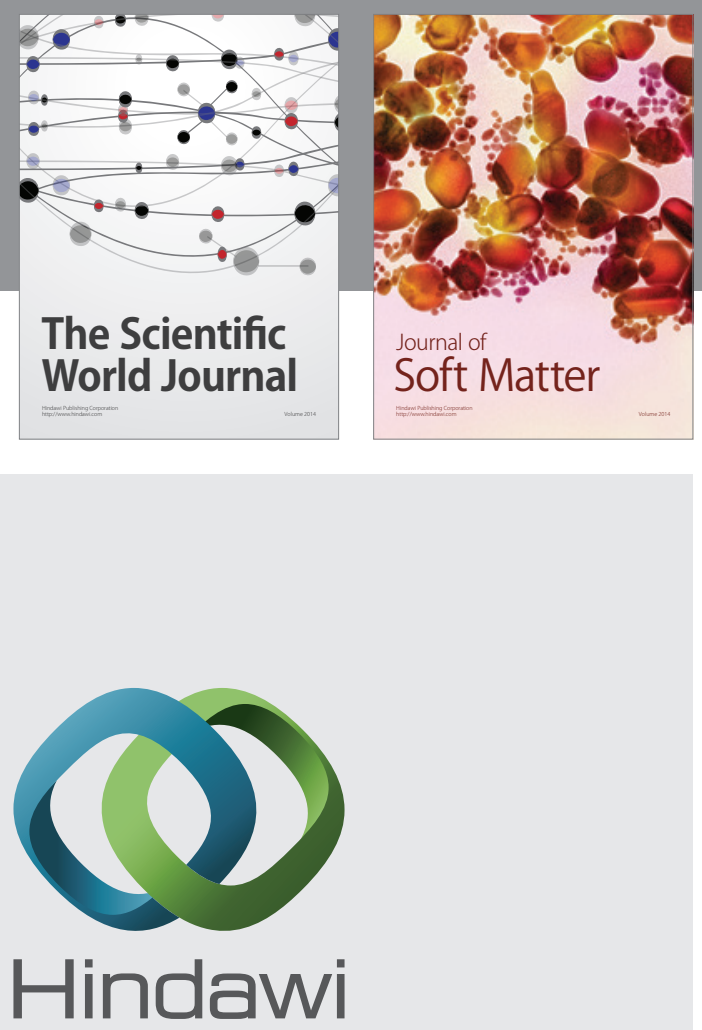

Submit your manuscripts at

http://www.hindawi.com

nternational Journal of

Statistical Mechanics
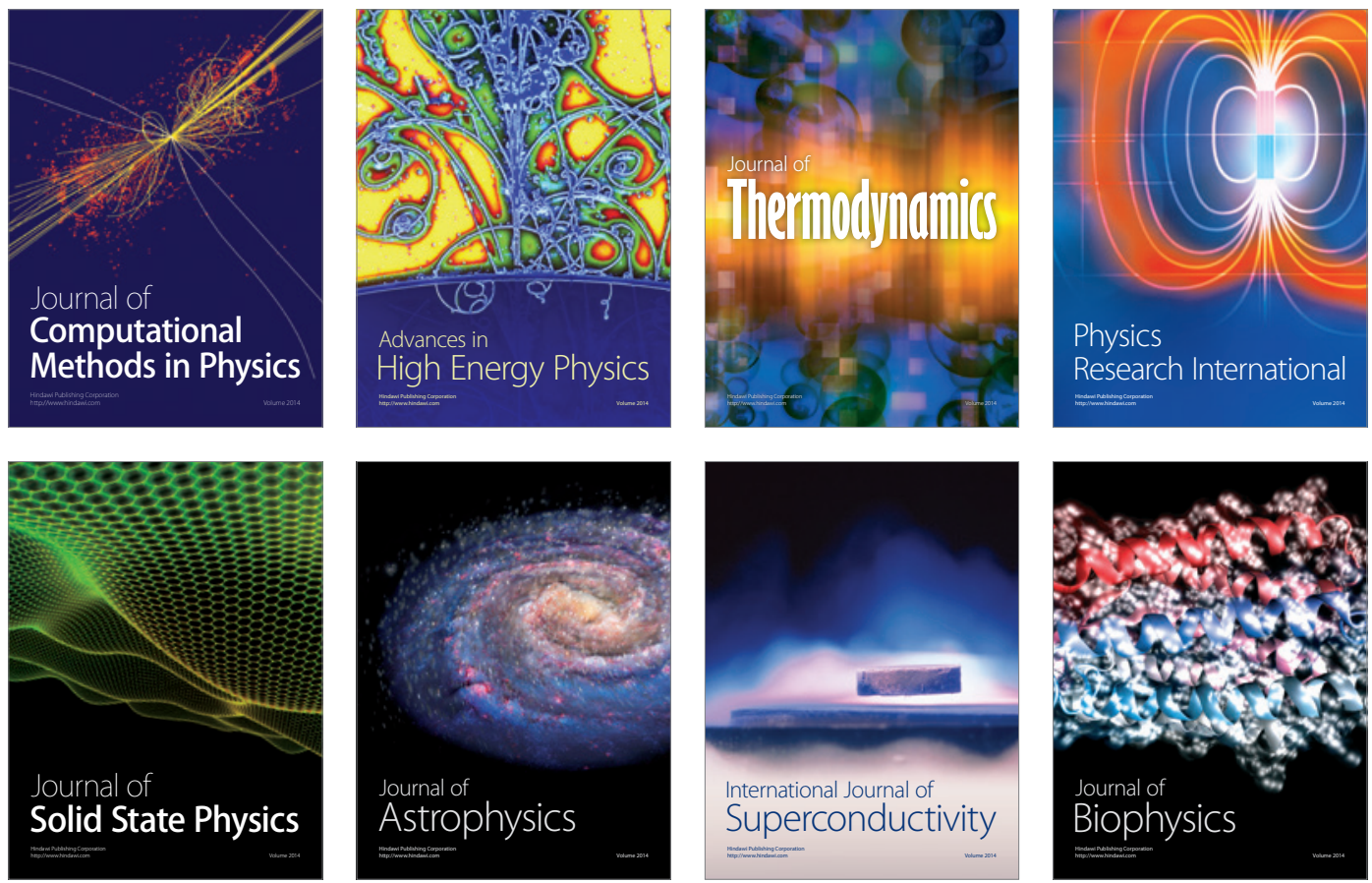
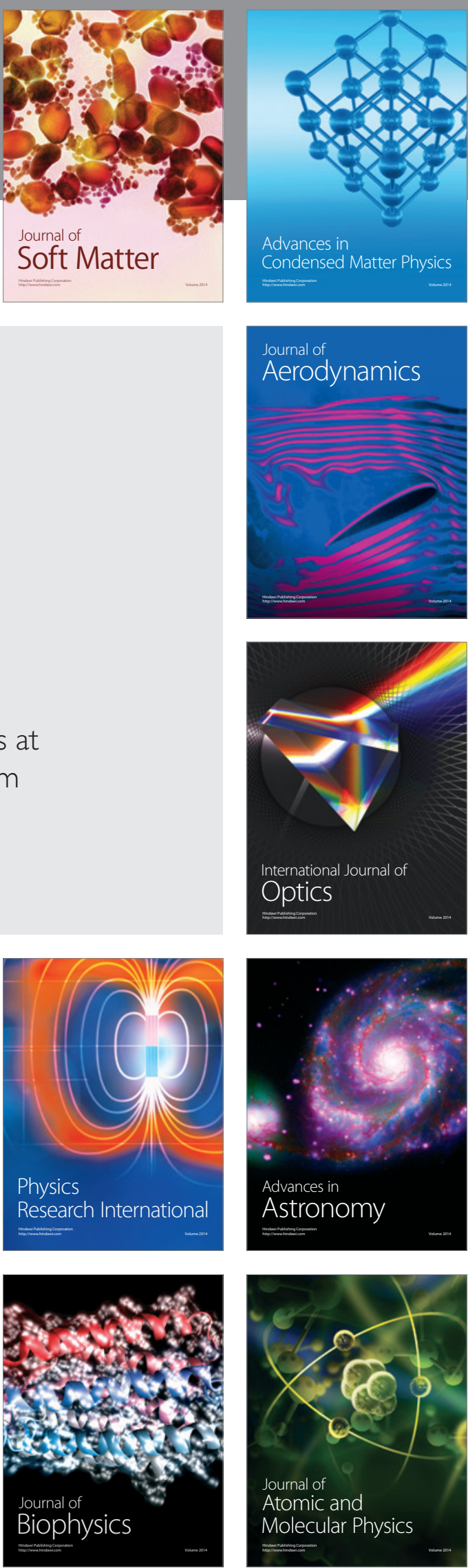